\title{
CONJUGACY IN GROUPS WITH DIHEDRAL 3-NORMALISERS
}

\author{
by N. K. DICKSON
}

(Received 18 March, 1976)

1. Introduction. Much work has been carried out on the classification of finite simple groups in terms of the structures of centralisers of involutions. However, it is sometimes the case that these classification results cannot be applied to particular problems even although information is available about one conjugacy class of involutions. The trouble is that information about the other classes can be almost non-existent. In this paper we deal with a situation where character theory can be employed to give a strong connection between the orders of centralisers of different classes of involutions, enabling information about one class to be used to give information about other classes. We prove the following result.

THEOREM 1. Let $G$ be a finite non-abelian simple group. Suppose that dis an element of $G$ of order 3 such that $N_{G}(\langle d\rangle)$ is dihedral. Then:

(a) every involution of $G$ is conjugate to an involution inverting $d$;

(b) if $\left|C_{G}(d)\right|$ is odd, $G$ has one conjugacy class of involutions;

(c) if $\left|C_{G}(d)\right|$ is even, $G$ has at most two conjugacy classes of involutions;

(d) if $\sigma$ and $\tau$ are representatives of different conjugacy classes of involutions, then $\left|C_{G}(\sigma)\right| /\left|C_{G}(\tau)\right| \leqq 2$ or $\left|C_{G}(\sigma)\right| /\left|C_{G}(\tau)\right|=(2 t+1) / t$ for some positive integer $t$.

REMARKS. 1. Note that part (d) implies that $\left|C_{G}(\sigma)\right| /\left|C_{G}(\tau)\right| \leqq 3$. Interchanging $\sigma$ and $\tau$, we also obtain: $\frac{1}{3} \leqq\left|C_{G}(\sigma)\right| /\left|C_{G}(\tau)\right| \leqq 3$.

2. Part (b) is already well known and is due to Brauer.

3. In Section 5 of this paper we discuss ways in which the hypotheses of Theorem 1 can be weakened.

Notation. We use the standard group theory notation of Gorenstein's book [2]; we will also adopt the convention of writing $N(H)$ and $C(H)$ for $N_{G}(H)$ and $C_{G}(H)$. For character theory we will refer to Dornhoff's book [1] and, following G. Higman [3], for $x, y, z \in G$ we let

$$
\#\left(x^{\cdot} y^{\cdot}=z\right)=\frac{|G|}{|C(x)||C(y)|} \sum \frac{\chi(x) \chi(y) \overline{\chi(z)}}{\chi(1)},
$$

where the summation is taken over a complete set of inequivalent ordinary irreducible characters $\chi$ of $G$. \# $\left(x^{\circ} y^{\circ}=z\right)$ is the number of ways $z$ can be written as a conjugate of $x$ times a conjugate of $y$. If $B$ is a block of $G, \#\left(x^{*} y^{*}=z\right)_{B}$ will denote the same formula as above except that the summation is restricted to characters in $B$.

Let $G$ and $d$ satisfy the hypotheses of Theorem 1. Let $N=N(\langle d\rangle), H=C(d), H_{0}$ $=O_{3},(H), \tau \in N \backslash H$, and let $b$ and $x$ generate a Sylow 3-subgroup and a Sylow 2-subgroup of $H$, respectively. (Note that we might have $x=1$.) Suppose that $|b|=3^{m}$ and $|x|=2^{n}$.

If $\phi$ is an ordinary irreducible character of $H_{0}$ let $E_{\phi}=\left\{g \in N: \phi^{g}(h)=\phi(h)\right.$ for all $\left.h \in H_{0}\right\}$. Since $H$ is abelian, $H \leqq E_{\phi}$ so that we may let $e_{\phi}=\left|E_{\phi}: H\right|$. Let $\phi_{0}$ be the principal

Glasgow Math. J. 18 (1977) 167-173. 
character of $H_{0}$ and, when $|H|$ is even, let $\phi_{1}$ be the character of $H_{0}$ such that $\left|H_{0}: \operatorname{ker} \phi_{1}\right|=2$ and $\phi_{1}(x)=-1$.

Let $\theta$ be a primitive $3^{m}$-th root of unity and let $\lambda_{i}, i=1, \ldots, 3^{m}-1$ be the non-principal irreducible characters of $\langle b\rangle$ given by $\lambda_{i}\left(b^{j}\right)=\theta^{i j}, j=0, \ldots, 3^{m}-1$.

2. Block structure of $G$. We note first that since $N(\langle d\rangle)$ is dihedral $\langle b\rangle$ is a Sylow 3subgroup of $G$ and that if $P$ is any 3-group containing $d$ then $N(P)=N(\langle d\rangle)=N$ and $C(P)=C(d)=H$.

A theorem of Dade (see [1, p. 420]) enables us to determine completely the 3-block structure of $G$. For ease of reference, we state those parts of Dade's result which we require.

LEMMA 2.1 (Dade). Let $\phi$ be an ordinary irreducible character of $H_{0}$, let $e=e_{\phi}$ and let $\Lambda$ be a set of representatives of the equivalence classes of non-principal ordinary irreducible characters of $\langle b\rangle$ under the action of $E_{\phi}$, so that $|\Lambda|=\left(3^{m}-1\right) / e$.

Then there is a 1-1 correspondence between 3-blocks of $G$ with defect $m$ and equivalence classes of ordinary irreducible characters of $H_{0}$ under the action of $N$ and, if $B$ is the block associated with the class containing $\phi$ :

(a) B contains $e$ irreducible Brauer characters $\psi_{1}, \psi_{2}, \ldots, \psi_{e}$ and $e+\left(3^{m}-1\right) / e$ ordinary irreducible characters $\chi_{1}, \chi_{2}, \ldots, \chi_{e}$ (the "non-exceptional" characters) and $\left\{\chi_{\lambda}: \lambda \in \Lambda\right\}$ (the " exceptional" characters);

(b) if notation is chosen so that $\chi=\sum_{i=1}^{e} d_{\chi, i} \psi_{i}$ on $3^{\prime}$-elements then

(i) all decomposition numbers in $B$ are 0 or 1 ,

(ii) $d_{x_{\lambda}, i}$ is independent of $\lambda$,

(iii) if $d_{\chi_{\lambda}, i}=0$ then $d_{x_{j}, i}=1$ for exactly two values of $j \in\{1, \ldots, e\}$ and if $d_{x_{\lambda}, i}=1$ then $d_{x j, i}=1$ for exactly one value of $j \in\{1, \ldots, e\}$ (in particular if $e=1$ then $\left.d_{x_{\lambda, 1}}=d_{\chi_{1}, 1}=1\right)$;

(c) there exist $\delta_{0}, \delta_{1}, \ldots, \delta_{e}, \gamma_{0}, \gamma_{1}, \ldots, \gamma_{3^{m-1}}$, each equal to \pm 1 , such that the generalised decomposition numbers are given by the following formulae (where $z \in N$ ):

$$
\begin{aligned}
& d_{\chi_{i}, \phi^{z}}^{b J}=\delta_{i} \gamma_{j}, \\
& d_{\chi_{\lambda, \phi^{z}}}^{b J}=\frac{\delta_{0} \gamma_{j}}{|H|} \sum_{y \in E_{\phi}} \lambda^{z y}\left(b^{j}\right) .
\end{aligned}
$$

LEMMA 2.2. If $\phi$ is an ordinary irreducible character of $H_{0}$ then $e_{\phi}=1$ except when $\phi=\phi_{0}$ or $\phi=\phi_{1}$ (and then $e_{\phi}=2$ ).

Proof. Since $H$ is abelian, $\phi^{h}=\phi$ and $\phi^{h \tau}=\phi^{\tau}$ for all $h \in H$. Thus $e_{\phi}=2$ if and only if $\phi=\phi^{\mathfrak{t}}$. But $\phi=\phi^{\mathrm{t}}$ if and only if $\phi(h)=\phi\left(\tau^{-1} h \tau\right)=\phi\left(h^{-1}\right)$ for all $h \in H_{0}$, i.e. if and only if $\phi\left(h^{2}\right)=1$ for all $h \in H_{0}$ (since $\phi$ is linear), i.e. if and only if $h^{2}$ is in the kernel of $\phi$ for all $h \in H_{0}$. The lemma follows immediately.

LEMMA 2.3. Let $\phi$ be an ordinary irreducible character of $H_{0}$, where $\phi \neq \phi_{0}, \phi_{1}$, and let $B$ be the block of $G$ with defect group $\langle b\rangle$ associated with $\phi$. Then: 
(a) $B$ contains $3^{m}$ ordinary irreducible characters, $\chi_{i}, i=0, \ldots, 3^{m}-1$, say,

(b) $\chi_{i}(k)$ is independent of $i$, for any $3^{\prime}$-element $k$,

(c) the notation can be chosen so that for all $h \in H_{0} \quad \chi_{i}\left(b^{j} h\right)=\varepsilon\left[\theta^{i j} \phi(h)+\theta^{-i j} \phi^{i}(h)\right]$ for $j=1, \ldots, 3^{m}-1$, where $\varepsilon= \pm 1$ and $\varepsilon$ is independent of $i$ and $h$ (but not necessarily of $j$ ).

Proof. By Lemma 2.2, $e_{\phi}=1$ and $E_{\phi}=H$. By Lemma 2.1, parts (a) and (b), parts (a) and (b) of this lemma are clear. Choosing notation so that $\chi_{0}$ is the non-exceptional character, $\chi_{0}\left(b^{j} h\right)=\varepsilon^{*}\left(\phi(h)+\phi^{\tau}(h)\right)$ for some $\varepsilon^{*}= \pm 1$, by Lemma 2.1, part (c). We can also choose notation so that there exists $\varepsilon= \pm 1$ such that

$$
\begin{aligned}
\chi_{i}\left(b^{j} h\right) & =\frac{\varepsilon}{|H|}\left[\left(\sum_{y \in H} \lambda_{i}^{y}\left(b^{j}\right)\right) \phi(h)+\left(\sum_{y \in H} \lambda_{i}^{\tau y}\left(b^{j}\right)\right) \phi^{\tau}(h)\right] \\
& =\frac{\varepsilon}{|H|}\left[\left(\sum_{y \in H} \theta^{i j}\right) \phi(h)+\left(\sum_{y \in H} \theta^{-i j}\right) \phi^{\tau}(h)\right] \\
& =\varepsilon\left[\theta^{i j} \phi(h)+\theta^{-i j} \phi^{\tau}(h)\right]
\end{aligned}
$$

for all $h \in H_{0}$ and $j \in\left\{1, \ldots, 3^{m}-1\right\}$.

Thus all that remains to be proved is that $\varepsilon^{*}=\varepsilon$. Since $\theta$ is a primitive $3^{m}$-th root of unity, $\sum_{i=1}^{3^{m}-1} \theta^{i j}=-1$. But the columns 1 and $b^{j} \operatorname{must}_{3^{m-1}}$ be orthogonal $\left(\right.$ i.e. $\left.\sum_{i=0}^{3 m-1} \chi_{i}(1) \chi_{i}\left(b^{j}\right)=0\right)$. By part (b), $\chi_{i}(1)$ is independent of $i$. Therefore $\sum_{i=0}^{m} \chi_{i}\left(b^{j}\right)=0$, which gives $\varepsilon^{*}=\varepsilon$.

LEMma 2.4. If $\phi_{1}$ exists, let $B_{1}$ be the block of $G$ with defect group $\langle b\rangle$ associated with $\phi_{1}$. Then $B_{1}$ has $\frac{1}{2}\left(3^{m}+1\right)$ ordinary irreducible characters $\zeta_{\alpha}, \zeta_{\beta}, \zeta_{i}, i=1,2, \ldots, \frac{1}{2}\left(3^{m}-1\right)$ such that the following is a fragment of the character table of $G$ :

\begin{tabular}{c|ccc} 
& 1 & $k$ & $b^{j} h$ \\
\hline$\zeta_{\alpha}$ & $\alpha_{1}$ & $\alpha_{k}$ & $\varepsilon_{1} \phi_{1}(h)$ \\
$\zeta_{\beta}$ & $\beta_{1}$ & $\beta_{k}$ & $\varepsilon_{2} \phi_{1}(h)$ \\
\hline$\zeta_{l}$ & $\varepsilon_{1} \varepsilon_{2} \alpha_{1}+\beta_{1}$ & $\varepsilon_{1} \varepsilon_{2} \alpha_{k}+\beta_{k}$ & $\varepsilon_{2} \phi_{1}(h)\left(\theta^{i j}+\theta^{-i j}\right)$
\end{tabular}

where $\alpha_{1} \leqq \beta_{1}, \alpha_{1}, \beta_{1}, \alpha_{k}, \beta_{k}$ are constants, $\varepsilon_{1}$ and $\varepsilon_{2}$ (which are independent of $h$ and $k$ but not necessarily of $j$ ) are $\pm 1, k$ is a $3^{\prime}$-element and $h \in H_{0}$.

Proof. By Lemma 2.2, $\phi_{1}=\phi_{1}{ }^{\tau}, e_{\phi_{1}}=2$ and $E_{\phi_{1}}=N$. By Lemma 2.1, part (a), we may let $\zeta_{\alpha}, \zeta_{\beta}$ be the non-exceptional characters and $\zeta_{i}, i=1, \ldots, \frac{1}{2}\left(3^{m}-1\right)$ be the exceptional characters. From Lemma 2.1, part (c), the rows of the character table corresponding to $\zeta_{\alpha}$ and $\zeta_{\beta}$ are as stated in the lemma. By Lemma 2.1, part (b), the value of $\zeta_{i}$ on $3^{\prime}$-elements is independent of $i$ and by part (c) we may choose $\varepsilon^{* *}= \pm 1, \varepsilon^{* *}$ independent of $i$, such that 


$$
\begin{aligned}
\zeta_{i}\left(b^{j} h\right) & =\frac{\varepsilon^{* *}}{|H|}\left(\sum_{y \in N} \lambda_{i}^{y}\left(b^{j}\right)\right) \phi_{1}(h) \\
& =\frac{\varepsilon^{* *}}{|H|}\left(\sum_{y \in H} \lambda_{i}^{y}\left(b^{j}\right)+\sum_{y \in H} \lambda_{i}^{y \tau}\left(b^{j}\right)\right) \phi_{1}(h) \\
& =\frac{\varepsilon^{* *}}{|H|}\left(\sum_{y \in H} \lambda_{i}\left(b^{j}\right)+\sum_{y \in H} \lambda_{i}^{\tau}\left(b^{j}\right)\right) \phi_{1}(h) \\
& =\varepsilon^{* *}\left(\theta^{i j}+\theta^{-i j}\right) \phi_{1}(h),
\end{aligned}
$$

noting that, since $E_{\phi_{1}}=N,\left\{\lambda_{i}: i=1, \ldots, \frac{1}{2}\left(3^{m}-1\right)\right\}$ is a set of representatives of the equivalence classes of non-principal ordinary irreducible characters of $\langle b\rangle$ under the action of $E_{\phi_{1}}$.

Orthogonality of the columns 1 and $b^{j}$ now gives $\varepsilon_{1} \alpha_{1}+\varepsilon_{2} \beta_{1}-\varepsilon^{* *} \zeta_{i}(1)=0$ since $\zeta_{i}(1)$ is independent of $i$. Thus $\varepsilon_{1} \varepsilon_{2} \alpha_{1}+\beta_{1}=\varepsilon_{2} \varepsilon^{* *} \zeta_{i}(1)$. Since $\alpha_{1} \leqq \beta_{1}$ and $\zeta_{i}(1)>0, \varepsilon_{2}=\varepsilon^{* *}$ and $\zeta_{i}(1)=\varepsilon_{1} \varepsilon_{2} \alpha_{1}+\beta_{1}$. Orthogonality of the columns $k$ and $b^{j}$ enables the remaining entries to be filled in.

LEMMA 2.5. $B_{0}$, the principal block of $G$, has $\frac{1}{2}\left(3^{m}+1\right)$ ordinary irreducible characters $\Phi_{0}, \Phi_{\gamma}, \Phi_{i}, i=1,2, \ldots, \frac{1}{2}\left(3^{m}-1\right)$ such that the following is a fragment of the character table of $G$ :

\begin{tabular}{c|ccc} 
& 1 & $k$ & $b^{j} h$ \\
\hline$\Phi_{0}$ & 1 & 1 & 1 \\
$\Phi_{\gamma}$ & $\gamma_{1}$ & $\gamma_{k}$ & $\varepsilon_{3}$ \\
\hline$\Phi_{i}$ & $\gamma_{1}+\varepsilon_{3}$ & $\gamma_{k}+\varepsilon_{3}$ & $\varepsilon_{3}\left(\theta^{i j}+\theta^{-i j}\right)$
\end{tabular}

where $\gamma_{1}, \gamma_{k}$ are constants, $\varepsilon_{3}$ (which is independent of $h$ and $k$ but not necessarily of $j$ ) is $\pm 1, k$ is $a$ $3^{\prime}$-element and $h \in H_{0}$.

Proof. The lemma follows immediately from the proof of Lemma 2.4 since $B_{0}$ is the block corresponding to $\phi_{0}$.

3. Conjugacy of involutions. We will now use the information obtained in Section 2 to prove Theorem 1, parts (a), (b) and (c).

Lemma 3.1. Let $k$ and $l$ be involutions of $G, h \in H_{0}$ and $j$ be such that $b^{j}=d$. Then we have the following table.

\begin{tabular}{c|c} 
3-Block $B$ & $\#\left(k \cdot l^{\cdot}=d h\right)_{B}$ \\
\hline$B_{0}$ & $\frac{|G|}{|C(k) \| C(l)|} \cdot \frac{\left(\gamma_{1}-\gamma_{k}\right)\left(\gamma_{1}-\gamma_{l}\right)}{\gamma_{1}\left(\gamma_{1}+\varepsilon_{3}\right)}$ \\
$B_{1}$ & $\frac{|G| \varepsilon_{1} \phi_{1}(h)}{|C(k) \| C(l)|} \cdot \frac{\left(\alpha_{1} \beta_{k}-\alpha_{k} \beta_{1}\right)\left(\alpha_{1} \beta_{1}-\alpha_{1} \beta_{1}\right)}{\alpha_{1} \beta_{1}\left(\varepsilon_{1} \varepsilon_{2} \alpha_{1}+\beta_{1}\right)}$ \\
$B \neq B_{0}, B_{1}$ & 0
\end{tabular}


Proof. If $B$ is a block of $G$ which does not have defect group $\langle b\rangle$ then, since the defect group of a block is an intersection of Sylow subgroups, $B$ has defect zero. So all characters of $B$ take value 0 on 3 -singular elements. Thus $\#\left(k^{*} \cdot l^{\cdot}=d h\right)_{B}=0$.

If $B$ is a block of the type dealt with in Lemma $2.3, \#\left(k^{\cdot} l^{\cdot}=d h\right)_{B}=0$ since $\sum_{i=1}^{3 m-1} \theta^{i j}=-1$.

The contributions from $B_{0}$ and $B_{1}$ follow from Lemmas 2.4 and 2.5.

LemMa 3.2. Let $k$ be any involution of $G$. Then $k$ is conjugate to an involution inverting $d$.

Proof. If $|H|$ is odd, then $\phi_{1}$ and $B_{1}$ do not exist and, from Lemma 3.1, \# $\left(k^{\circ} k^{\circ}=d\right)=$ $\#\left(k^{*} k^{\cdot}=d\right)_{B_{0}} \neq 0$ because the simplicity of $G$ forces $\gamma_{k} \neq \gamma_{1}$.

If $|H|$ is even then, noting that $\phi_{1}(x)=-1$, we see from Lemma 3.1 that we may write

$$
\begin{gathered}
\#\left(k^{\cdot} k^{\cdot}=d\right)=\#\left(k^{\cdot} k^{\cdot}=d\right)_{B_{0}}+\#\left(k^{\cdot} k^{\cdot}=d\right)_{B_{1}} \\
\text { and } \\
\#\left(k^{\cdot} k^{\cdot}=d x\right)=\#\left(k^{\cdot} k^{\cdot}=d\right)_{B_{0}}-\#\left(k^{\cdot} k^{\cdot}=d\right)_{B_{1}} .
\end{gathered}
$$

As remarked above $\#\left(k^{\circ} k^{\circ}=d\right)_{B_{0}} \neq 0$. Therefore at least one of $\#\left(k^{\circ} k^{\circ}=d\right)$ and $\#\left(k^{\cdot} k^{\cdot}=d x\right)$ is non-zero.

So in all cases $k$ has two conjugates which generate a dihedral group containing $d$, i.e. some conjugate of $k$ inverts $d$.

LEMMA 3.3. If $|H|$ is odd, $G$ has one conjugacy class of involutions; if $|H|$ is even, $G$ has at most two classes of involutions.

Proof. $N(\langle d\rangle)$ is dihedral and therefore has one conjugacy class of involutions inverting $d$ if $|H|$ is odd and two such classes if $|H|$ is even. The lemma now follows from Lemma 3.2.

4. The case when $G$ has two classes of involutions. In this section we prove part (d) of Theorem 1. Suppose that $G$ has two classes of involutions. By Lemma 3.3 $|H|$ is even and we may choose our notation so that $\tau$ is a representative of any specified class. Then $x \tau$ is a representative of the other class. Let $\sigma=x \tau$.

LemMa 4.1 .

$$
\begin{aligned}
& \#\left(\sigma^{\circ} \sigma^{\cdot}=d\right)=\#\left(\sigma^{\circ} \tau^{\cdot}=d x\right)=\frac{1}{2}|H| \\
& \quad \text { and } \\
& \#\left(\sigma^{\circ} \tau^{\cdot}=d\right)=\#\left(\sigma^{\circ} \sigma^{\cdot}=d x\right)=0 .
\end{aligned}
$$

Proof. Since all involutions of a dihedral group of order 6 are conjugate, \# $\left(\sigma^{\circ} \tau^{\circ}=d\right)=0$. $N(\langle x, \tau\rangle) \cap N(\langle d\rangle)=\langle x, \tau\rangle$ so that $N$ contains $|N| / 2^{n+1}=|H| / 2^{n}$ Sylow 2-subgroups (all containing $x$ ). Since a dihedral group of order $3.2^{n+1}$ contains 3 Sylow 2-subgroups, $N$ has $|H| / 3.2^{n}$ dihedral subgroups of order $3.2^{n+1}$ (all containing $d x$ ).

In the dihedral group $\langle d, x, \tau\rangle$ of order $3.2^{n+1}$ the only ordered pairs $(k, l)$ of involutions with $k l=d x$ are given by $k=(d x)^{i} \tau$ and $l=(d x)^{i-1} \tau, 0 \leqq i \leqq 3.2^{n}-1$. Since for all $j,(d x)^{2 j} \tau$ is conjugate to $\tau$ and $(d x)^{2 j+1} \tau$ is conjugate to $x \tau$, it follows that the contributions to $\#\left(\sigma^{\circ} \sigma^{\circ}=\right.$ $d x)$ and $\#\left(\sigma^{\circ} \tau^{\circ}=d x\right)$ are 0 and $3.2^{n-1}$ respectively. So $\#\left(\sigma^{\circ} \sigma^{\circ}=d x\right)=0$ and $\#\left(\sigma^{\circ} \tau^{\circ}=d x\right)$ $=3.2^{n-1} \cdot|H| / 3.2^{n}=\frac{1}{2}|H|$. 
Now $\langle d, x, \tau\rangle$ contains $2^{n}$ dihedral subgroups of order 6 , namely $\left\langle d,(d x)^{i} \tau\right\rangle, 0 \leqq i \leqq 3.2^{n}-1$. Of these $2^{n-1}$ have involutions conjugate to $\sigma$ and $2^{n-1}$ have involutions conjugate to $\tau$. Thus $N$ contains $2^{n-1} \cdot|H| / 3.2^{n}=\frac{1}{6}|H|$ dihedral groups of order 6 with involutions conjugate to $\sigma$. Therefore $\#\left(\sigma^{\circ} \sigma^{\circ}=d\right)=3 \cdot \frac{1}{6}|H|=\frac{1}{2}|H|$, as required.

LeMma 4.2.

$$
|C(\sigma)| /|C(\tau)|=\left(\gamma_{1}-\gamma_{\sigma}\right) /\left(\gamma_{1}-\gamma_{t}\right) \text {. }
$$

Proof. By Lemmas 3.1 and 4.1, since $\phi_{1}(x)=-1$,

and

$$
\begin{aligned}
\frac{1}{4}|H| & =\frac{1}{2}\left[\#\left(\sigma^{\circ} \sigma^{\circ}=d\right)\right]+\frac{1}{2}\left[\#\left(\sigma^{\circ} \sigma^{\circ}=d x\right)\right] \\
& =\frac{|G|}{|C(\sigma)|^{2}} \cdot \frac{\left(\gamma_{1}-\gamma_{\sigma}\right)^{2}}{\gamma_{1}\left(\gamma_{1}+\varepsilon_{3}\right)}
\end{aligned}
$$

whence the result.

$$
\begin{aligned}
\frac{1}{4}|H| & =\frac{1}{2}\left[\#\left(\sigma^{\circ} \tau^{\circ}=d\right)\right]+\frac{1}{2}\left[\#\left(\sigma^{\circ} \tau^{\circ}=d x\right]\right. \\
& =\frac{|G|}{|C(\sigma) \| C(\tau)|} \cdot \frac{\left(\gamma_{1}-\gamma_{\sigma}\right)\left(\gamma_{1}-\gamma_{\tau}\right)}{\gamma_{1}\left(\gamma+\varepsilon_{3}\right)},
\end{aligned}
$$

REMARK. The proof of the next lemma is the first place where we use in an essential way the fact that $d$ has order three rather than any odd prime.

LEMMA 4.3.

$$
\frac{\gamma_{1}-\gamma_{\sigma}}{\gamma_{1}-\gamma_{\tau}} \leqq 2+\frac{2 \varepsilon_{3}}{\gamma_{1}-\gamma_{\tau}}
$$

Proof. Let $M$ be the module affording $\Phi_{\gamma}$. ( $\Phi_{\gamma}$ is defined in Lemma 2.5.) Then $M$ is completely reducible as a $\langle d, \tau\rangle$-module so that we may suppose that $d$ and $\tau$ are represented by matrices of the form

$$
\operatorname{diag} \underbrace{\left(1,1, \ldots, 1, \eta, \eta^{-1}, \ldots, \eta, \eta^{-1}\right)}_{r \text { entries }}
$$

and

$$
\operatorname{diag}\left( \pm 1, \pm 1, \ldots, \pm 1,\left[\begin{array}{ll}
0 & 1 \\
1 & 0
\end{array}\right], \ldots,\left[\begin{array}{ll}
0 & 1 \\
1 & 0
\end{array}\right]\right)
$$

respectively, where $\eta$ is a primitive cube root of unity. Clearly $\left|\gamma_{\tau}\right|=\left|\Phi_{\gamma}(\tau)\right| \leqq r$. Since $\eta+\eta^{-1}=-1, \Phi_{\gamma}(d)=r-\frac{1}{2}\left(\Phi_{\gamma}(1)-r\right)$, i.e. $r=\frac{1}{3}\left(\Phi_{\gamma}(1)+2 \Phi_{\gamma}(d)\right)=\frac{1}{3}\left(\gamma_{1}+2 \varepsilon_{3}\right)$.

Thus $\left|\gamma_{\tau}\right| \leqq \frac{1}{3}\left(\gamma_{1}+2 \varepsilon_{3}\right)$. By considering $\langle d, \sigma\rangle$ we also obtain $\left|\gamma_{\sigma}\right| \leqq \frac{1}{3}\left(\gamma_{1}+2 \varepsilon_{3}\right)$.

Now $2 \gamma_{\tau}-\gamma_{\sigma} \leqq 2\left|\gamma_{\tau}\right|+\left|\gamma_{\sigma}\right| \leqq \gamma_{1}+2 \varepsilon_{3}$ so that $\gamma_{1}-\gamma_{\sigma} \leqq 2\left(\gamma_{1}-\gamma_{\tau}\right)+2 \varepsilon_{3}$. In addition $\gamma_{1}-\gamma_{\mathrm{r}}$ is a positive even integer (because it equals twice the number of times $\tau$ has -1 as an eigenvalue). Thus

$$
\frac{\gamma_{1}-\gamma_{\sigma}}{\gamma_{1}-\gamma_{\tau}} \leqq 2+\frac{2 \varepsilon_{3}}{\gamma_{1}-\gamma_{\tau}}, \text { as required. }
$$

LeMmA 4.4. $|C(\sigma)| /|C(\tau)| \leqq 2$ or $|C(\sigma)| /|C(\tau)|=(2 t+1) / t$ for some positive integer $t$. 
Proof. By Lemma 4.2 it is sufficient to prove that $\left(\gamma_{1}-\gamma_{\sigma}\right) /\left(\gamma_{1}-\gamma_{\tau}\right) \leqq 2$ or $\left(\gamma_{1}-\gamma_{\sigma}\right) /$ $\left(\gamma_{1}-\gamma_{t}\right)=(2 t+1) / t$ for some integer $t$.

Suppose that $\left(\gamma_{1}-\gamma_{\sigma}\right) /\left(\gamma_{1}-\gamma_{\tau}\right)>2$. Since $\left(\gamma_{1}-\gamma_{\sigma}\right) /\left(\gamma_{1}-\gamma_{\tau}\right)$ is the ratio of two integers we may write it in the form $2+(s / t)$ where $s$ and $t$ are positive integers with no common factor. From Lemma $4.3 s / t \leqq 2 \varepsilon_{3} /\left(\gamma_{1}-\gamma_{\tau}\right)$ so that $\varepsilon_{3}=1$ and $s\left(\gamma_{1}-\gamma_{\imath}\right) \leqq 2 t$.

Now $\gamma_{1}-\gamma_{t}$ and $\gamma_{1}-\gamma_{\sigma}=(2+(s / t))\left(\gamma_{1}-\gamma_{\tau}\right)$ are positive even integers. Since $s$ and $t$ have no common factor, $t$ divides $\gamma_{1}-\gamma_{\tau}$. But, from above, $s\left(\gamma_{1}-\gamma_{\tau}\right) \leqq 2 t$. Therefore we either have $s=1$ (and $\gamma_{1}-\gamma_{\tau}=t$ or $\gamma_{1}-\gamma_{\tau}=2 t$ ) or $s=2$ (and $\gamma_{1}-\gamma_{\tau}=t$ ). In the latter case $t$ is now even and so $s$ and $t$ have 2 as a common factor. This contradiction establishes that $s=1$, which proves the lemma.

5. An extension. In Sections 3 and 4 we proved Theorem 1. An examination of this proof shows that only 2-subgroups and 3-subgroups of $G$ play an important role and that we have in fact obtained the following slightly stronger result.

THEOREM 2. Let $G$ be a finite non-abelian simple group. Suppose that dis an element of $G$ of order 3 such that

(i) $C(d)=H_{1} \times H_{2}$ where $H_{1}$ is abelian of order prime to 2 and 3 and $H_{2}$ is cyclic, and

(ii) $N(\langle d\rangle)=C(d) .\langle\tau\rangle$ for some element $\tau$ such that $\tau^{-1} h \tau=h^{-1}$ for all $h \in C(d)$.

Then $\tau^{2}=1$ and conclusions (a), (b), (c) and (d) of Theorem 1 hold.

(Note that the conclusion that $\tau^{2}=1$ arises as follows. Certainly $|N(\langle d\rangle): C(d)|=2$ since $d$ has order 3. So $\tau^{2} \in C(d)$. Lemma 3.2 proves that there are involutions inverting $d$, whence $\tau^{2}=1$.)

ACKNOWLEDGEMENTS. The contents of this paper are largely taken from the author's Oxford D.Phil. thesis. The author wishes to thank Dr W. B. Stewart, who supervised his work on that thesis, and also the Carnegie Trust for the Universities of Scotland, which provided financial support.

\section{REFERENCES}

1. L. Dornhoff, Group Representation Theory (2 vols.) (Dekker, 1971).

2. D. Gorenstein, Finite Groups (Harper and Row, 1968). 1968).

3. G. Higman, Odd characterisations of finite simple groups, Lecture Notes (University of Michigan,

\section{UNIVERSITY OF GLASGOW}

DePaRTMENT OF MATHEMatics

UNIVERSITY GARDENS

GLASGOW

SCOTLAND

G12 8QW 